\title{
Defended Data Embedding For Chiseler Avoidance in Visible Cryptography by Using Morphological Transform Domain
}

\author{
Nalla Bariki Praveen Kumar \\ CSE, MVGR Engineering College/ JNTU Kakinada,,INDIA
}

\begin{abstract}
This paper proposes a data-veiling technique for binate images in morphological transform domain for authen- tication purpose. To attain blind watermark drawing, it is difficult to use the detail coordinate precisely as location map to regulate the data-veiling locations. Thus, we look flipping an edge pixel in binate images as deviating the edge location one pixel horizontally, one vertically. Positioned on this conclusion, we propose an interlaced morphological binate wavelet transform to path the alter edges, which thus ease blind watermark drawing and fusion of cryptographic indication.Unlike current block-based approach, in that the block size is given as $3 \times 3$ pixels and larger, we establish the image in $2 \times 2$ pixel blocks. It allows resilience in discovering the edges and also gets the low computational complication. There are two case that twisting the candidates of one do not change the flippability circumstances of other are engaged for orthogonal embedding, that deliver more relevant candidates can be determined so that a larger quqntity can be accomplished.A contemporary effective Backward-Forward Minimization method is suggested, which acknowledge the backward i.e enclose candidates and forward those twisted candidates that may be concerned by spining the present pixel. By this way, the complete visual bias can be minimized. Experimental results determine the validity of our arguments.
\end{abstract}

Keywords: Verification, binate images, data cloaking, mor- phological binate wavelet transform, Quadrate embedding.

\section{INTRODUCTION}

Water marking and data-veiling techniques have found wide applications in ownership recognition,Copy protecting, finger printing,verification and.The pattern concern for a data-veiling and watermarking system are distinct catering for different applications. Now a days verification of digital documents has found great interest due to the different types of applications in normal signatures, digital books,documents, files,personal documents, maps,and so on.On the other hand, editing an image becomes very simple with the dominant image compileing tools and digital cameras. Verification to detect meddle and falsification is thus of primary thing. To provide the authenticity and integrity of the documents has expanded the confidence level from the user mark of view.many data-veiling techniques for binate images are based on dimensional, selecting data-veiling locations by applying pairs of contour edge patterns,edge pixels, visible distortion tables and illustrating visual quality- conserve rules. However, the abundance of the actual algorithms are not broad enough, exclusively for limited images. For example, the scope as large as 512 bits is required to incorporate a message verification code such as SHA-2. In addition, the existing large quantity algorithm does not have good visible quality of the watermarked image and the computational load is relatively big. Provoked by the above conclusion, we intend to design a high-capacity data-veiling scheme with low computational complexity and acceptable visual quality. We investigate the data veiling in transform domain to identify the data-veiling locations to evade the inconvient pattern-matching process. The goal of verification is to assure that a given set of data appear from a appropriate sender and the contented integrity is defended.Strong verification rejects any alteration made to a multimedia signal, whereas soft verification differs- entiates legitimate processing from malicious meddle. This paper targets on hard authenticator watermark-based verification. Specifically, we investigate the problem of data veiling for binate images in semantic transform domain. Commonly tallking, data veiling in real-valued alter concern does not perform well for binate images due to the quanti- zation failure imported in the pre/post-processing. In extension, enclose data using absolute-valued coefficients lack more memory space. We examine that the morphological binate wavelet alteration can be used to path the transitions in binate images by utilizing the design coefficients. One rather intu- itive idea in employing the morphological binate wavelet trans- form for data veiling is that it uses the design coefficients as a loca- tion point to determine the data-veiling locations. However, this makes it crucial to get the blind watermark extraction due to the case that once a pixel is twisted, the horizontal, vertical and diagonal detail coefficient will change appropriately. This design will be discussed in more detail further. The idea of conniving an interlaced alteration to identify the embeddable points is provoked by the fact that some conversion information is hidden during the computation of a single alteration and there is a demand to keep record of conversions between two and three pixels for binate images data veiling. Definitely, we process the images based on $2 \times 2$ 
pixel blocks and associate two distinct processing cases that the flippability conditions of one

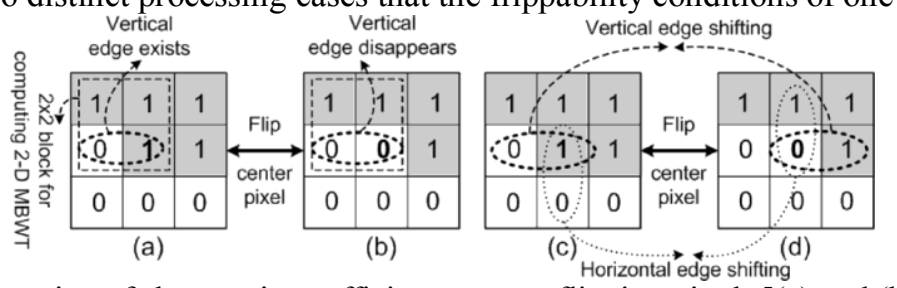

Fig. 1. Illustration of changes in coefficients owe to flipping pixels [(a) and (b)]

and the "edge shifting" phenomenon [(c) and (d)].

are not concerned by twisting the candidates of another for data em- bedding, namely "orthogonal embedding". This locates the diificulty of the quantity decrease due to the un embeddability of the section bounds, distribution rows and columns in block-based approaches.As a result, important gains in capacity can be accomplished, which also develops the efficiency of employing the flippable pixels. Acheiving the transforms by the "Exclusive OR" operation locates the quantization failure issue in a DCT-placed access. The extension advantages of the pro-posed scheme lie in its larger quantity,better visual quality and lower computational complexity. In extension, unlike of our present scheme does not experience the quqntity decrease and computational load increase in order to absorb the cryptographic signature for evidence. This paper is formulated as pursue. A brief review on current 1-D morphological binate wavelet transform (MBWT), our proposed expansion of 2D MBWT and interlaced MBWT (IMBWT) are shown in Section II.

\section{INTERLACED MORPHOLOGICAL TRANSFORM}

One innate idea in employing MBWT for data veiling is to use the design coefficients as a point map to regulate the data- veiling locations, since these coefficients contain the edge infor- mation in horizontal, vertical and diagonal directions. Flipping a pixel involves changing the coefficients, as described in above figure. It can be seen that the like edges used to regulate the data-veiling points cannot be seen in the watermarked image. We observe that flipping an edge pixel in binate images is identical to deviating the edge position horizontally one pixel and vertically one pixel. In above fig, " 1 " and " 0 " rep- resent the black and white pixels, appropriately.At the last, we develop an IMBWT to keep path of the alter edges to get blind watermark extraction.

In this part,we intiate the consideration with signal analysis and synthesis which is identical. A brief analysis on the 1D signal decomposition is also included. Based on this, we further extend the disintegration scheme to 2-D signal and finally propose an interlaced transform for the data-veiling function.

\section{MORPHOLOGICAL BINATE WAVELET TRANSFORM}

The first paragraph under each heading or subheading should be flush left, and subsequent paragraphs should have a five-space indentation. Consider a folk of signal area $\boldsymbol{\square}$ and detail space $\square$ at level . The 1-D wavelet disintegration pattern constiute of one signal analysis operator and one detail analyze operator

In extension, it also dwell of one signal synthesis operator $\varpi_{1}^{+}$and 1 detail synthesis operator $w_{c}$. The "+" denotes that the comparable operator maps a signal to a higher level, towards the direction of decreasing the information. Whereas " " defines that the operator model a signal to a lower level almost to the direction of backup the information. The signal analysis operator $\psi_{i}^{+}: V_{l} \rightarrow V_{l+1}$ map a signal,. So detail analysis operator $w_{t}^{+}=V_{\varepsilon} \longrightarrow \boldsymbol{W}_{z+1}$ map a signal from level $\ell$ to $\ell+1$ for the detail signal

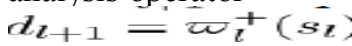

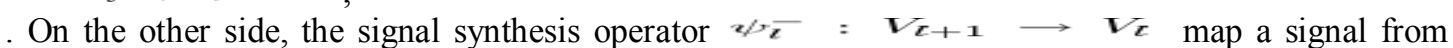

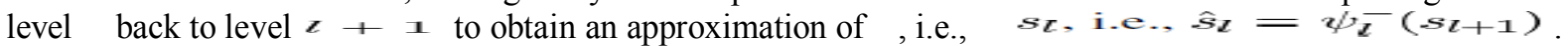

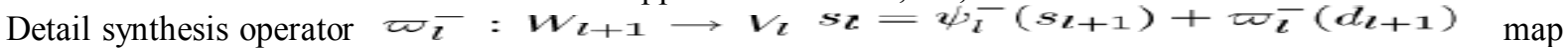
a detail signal back to level so as to get the detail signal $e_{\varepsilon}=\omega_{x}\left\langle\alpha_{x+1}\right\rangle$. The signal at level $Z$

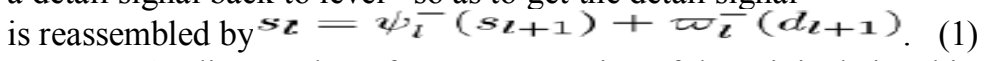

As discussed, perfect reconstruction of the original signal is feasible if the operators satisfy

$$
\begin{aligned}
& \psi_{l}^{-} \psi_{l}^{+}=\varpi_{l}^{-} \varpi_{l}^{+}=I \\
& \psi_{l}^{-} \varpi_{l}^{+}=\varpi_{l}^{-} \psi_{l}^{+}=\underline{0} \\
& \psi_{l}^{-} \psi_{l}^{+}+\varpi_{l}^{-} \varpi_{l}^{+}=I
\end{aligned}
$$

where "I" and "O" defines the integrity, zero operators respectively. Note that is a set of bi-orthogonal filter operators if the conditions in are contented. For morphological analysis and synthesis scheme demonstrated in this paper, the bi-orthogonality is defined in the operator terms. For 
directness, we shall delete the index 1 in the related analysis and synthesis operators owed to only one step decomposition between $\boldsymbol{V}_{0}$ and $\left\{\boldsymbol{V}_{\mathbf{1}}, \boldsymbol{W}_{\mathbf{1}}\right\}$ is considered. Let " $\oplus$ " denote the "Exclusive OR" operation. The signals at level $l+1$ by employing the analysis operators for a 1-D signal are given by

$$
\begin{aligned}
& s(i)=\psi^{+}(s)(i)=s(2 i+1) \\
& d(i)=\varpi^{+}(s)(i)=s(2 i) \oplus s(2 i+1)
\end{aligned}
$$

where $s(i)$ and $d(i)$ and are the coarse and design signals achieved at level $l+1$, respectively; denotes the basis of the signal at level $z+1$ and $i=0,1,2, \ldots, N-1$ for a 1 -D signal of size $\mathrm{N}$. The design signal $d(i) \quad$ insert 1 at each evolution from 0 to 1 or vice versa in signal . The synthesis operators are given by

$$
\begin{aligned}
& \psi^{-}(s)(2 i+1)=\psi^{-}(s)(2 i)=s(i) \\
& \varpi^{-}(s)(2 i+1)=0 \text { and } \varpi^{-}(s)(2 i)=d(i) .
\end{aligned}
$$

The signal at level is reassembled by

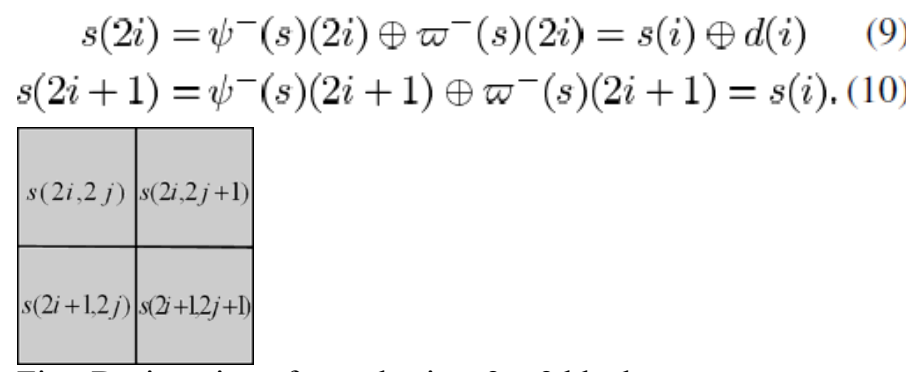

Fig: Designation of samples in a $2 \times 2$ block.

\section{B. 2-D and Interlaced Morphological Binate Wavelet Transform}

So now we prolong the 1D wavelet decomposition scheme to a $2 \mathrm{D}$ signal by describing a nonseparable 2D transform. Let and mean the ratio of the signal at level $l+1$, where $i=0,1,2, \ldots M-1$ and $j=0,1,2, \ldots N-1$ for a $2-\mathrm{D}$

Coarse signal size $\mathrm{MxN}$. Designation of the samples in a $2 \times 2$ block is shown above Fig, where $s(2 i, 2 j)$ denotes the signal (" 0 " or " $1 ")$ located at row $2 i$ and column $2 j$ at other level . To salient a 2-D transform, 1 sample in a 2 x 2 block can be subsampled as the coarse signal. The horizontal, vertical and diagonal design signals are derived from the distinction between the subsampled sample and the vertical, horizontal and diagonal acquaintance. The resultant transformed signal remains binate although the coarse and design signals will any be $1 / 4$ the size of the initial signal. The operators of the coarse signal, horizontal, vertical, and diagonal design signals be $\psi^{e e}, \omega_{h}^{e e}, \omega_{v}^{e e}$ and $\varpi_{d}^{e e}$ where the superscript "ce" denotes "even-even". The obtained trans-form is named the even- even transform since it is regulated on $l+12 \times 2$ block initiating from the even -even coordinates. Coarse signal, vertical, horizontal and diagonal design signals at level are obtained by employing the analysis operators to crop.

$$
\begin{aligned}
& s^{e e}(i, j)=i e^{e+1}(-s)(i, j)=s(2 i+1,2 j+1) \quad \text { (11) } \\
& v^{e e}(i, j)=\omega_{i}^{e+t}(s)(i, j)=s(2 i+1,2 j) \\
& \oplus s(2 i+1,2 j+1) \\
& h^{\text {ee }}(i, j)=\omega_{h}^{e+}(s)(i, j)=s(2 i, 2 j+1) \\
& \oplus s(2 i+1,2 j+1) \quad \text { (13) } \\
& d^{e e}(i, j)=\omega_{d}^{e+}(s)(i, j)=s(2 i, 2 j) \oplus s(2 i+1,2 j) \\
& \oplus s(2 i, 2 j+1) \oplus s(2 i+1,2 j+1) \quad \text { (14) } \\
& \text { where } \mathcal{s e e}^{e}(i, j) \in V_{i+1} \text { and }\left\{\operatorname{vee}^{e}(i, j), h^{e e}(i, j), d^{e e}(i, j)\right\} \in
\end{aligned}
$$

At last, the signal at level can be reassembled by 


$$
\begin{aligned}
s(2 i, 2 j)= & \psi^{e e}-(s)(2 i, 2 j) \oplus \varpi_{d}^{e e}(s)(2 i, 2 j) \\
= & s^{e e}(i, j) \oplus v^{e e}(i, j) \oplus h^{e e}(i, j) \\
& \oplus d^{e e}(i, j) \\
s(2 i, 2 j+1)= & \psi^{e e}(s)(2 i, 2 j+1) \oplus \varpi_{h}^{e e}-(s)(2 i, 2 j+1) \\
= & s^{e e}(i, j) \oplus h^{e e}(i, j) \\
s(2 i+1,2 j)= & \psi^{e e}(s)(2 i+1,2 j) \oplus \varpi_{v}^{e e}-(s)(2 i+1,2 j) \\
= & s^{e e}(i, j) \oplus v^{e e}(i, j) \\
s(2 i+1,2 j+1)= & \psi^{e e}-(s)(2 i+1,2 j+1) \\
& \oplus \varpi^{e e}-(s)(2 i+1,2 j+1)=s^{e e}(i, j) .
\end{aligned}
$$

The coarse signals accessed from 11 are at the odd- odd positions, the transitions from odd-odd to other coordinates in the $2 \times 2$ block may be easily determined from the detail signals obtained by eq 12-14. However, the development from odd even, even odd and even even coordinates to other coordinates may not be so easily determined. This provokes the design of complementary wavelet transforms operating on the $2 \times 2$ block starting from the even odd, odd even and odd-odd coordinates of the signal. Based on the initial complete coordinates in the top left position shown in Fig:ii each 2 x 2 block in a 2D image can be defined as an eveneven block EEB, or an even odd block EOB, or odd even block OEB, or an odd odd block OOB, which is given as

$$
B_{p}(i, j) \in \begin{cases}E E B s & \text { for }(\bmod (x, 2)=0) \wedge(\bmod (y, 2)=0) \\ E O B s & \text { for }(\bmod (x, 2)=0) \wedge(\bmod (y, 2)=1) \\ O E B s & \text { for }(\bmod (x, 2)=1) \wedge(\bmod (y, 2)=0) \\ O O B s & \text { for }(\bmod (x, 2)=1) \wedge(\bmod (y, 2)=1)\end{cases}
$$

where $(x, y)$ defines $x t h$ row and $y t h$ column of an image (i,j) defines the index of the current 2 x 2 block, denotes a modulo operation and " $"$ " represents logical "AND" operation. Thus, 3 more transforms even odd, odd even and odd odd, can thus described. These alterations with the even-even transform, are generally called interlaced morpho logical binate wavelet transform (IMBWT).

Let the operators of the even-odd transforms be $\left\{\psi^{e a}, \varpi_{h}^{\infty \infty}, \varpi_{v}^{e \bar{o}}, \varpi_{d}^{e a}\right\}$, the signal thus got by employing the analysis operators for the even-odd transforms are given as

$$
\begin{aligned}
s^{e o}(i, j)= & \psi^{e o+}(s)(i, j)=s(2 i+1,2 j+2) \\
v^{e o}(i, j)= & \varpi_{v}^{e o+}(s)(i, j)=s(2 i+1,2 j+1) \\
& \oplus s(2 i+1,2 j+2) \\
h^{e o}(i, j)= & \varpi_{h}^{e o+}(s)(i, j)=s(2 i, 2 j+2) \\
& \oplus s(2 i+1,2 j+2) \\
d^{e o}(i, j)= & \varpi_{d}^{e o+}(s)(i, j)=s(2 i, 2 j+1) \oplus s(2 i+1,2 j+1) \\
& \oplus s(2 i, 2 j+2) \oplus s(2 i+1,2 j+2) .
\end{aligned}
$$

The odd even , odd odd transforms can be described in this way. For simplicity, we use the

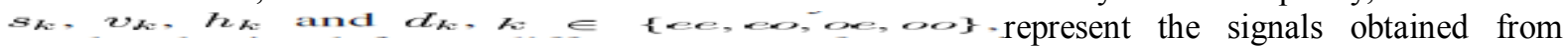
distinct alterations. There are 4 single processing even-even, even odd, odd even and odd -odd that are element on the main alter sections to be EEB, EOB, Where $\square$ is the odd-even feature of of . The extracted payload watermark is given by

$$
\begin{aligned}
\mathcal{W}_{s}^{\prime} \oplus h\left(Y_{w 1}\right) & =h^{\prime}\left(Y_{1}\right) \oplus \mathcal{W}_{p}^{\prime} \oplus h\left(Y_{w 1}\right) \\
& =\mathcal{W}_{p}^{\prime} \text { for } h^{\prime}\left(Y_{1}\right)=h\left(Y_{w 1}\right)
\end{aligned}
$$

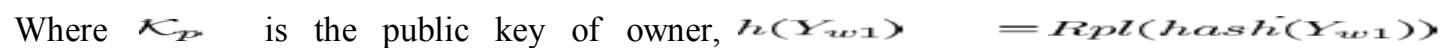
and $h^{\prime}\left(Y_{1}\right)$ is retrieved and replicated hash value of . Authenticity and integrity of $\boldsymbol{Y}_{\boldsymbol{w}}$ are determined by comparing the extracted $W_{p}^{\prime}$ with the original $W_{P}$. Noticeably, both flippable candidates should be cleared out to generate the transitional image using DPDC $\mathcal{D}_{b}(i, j)$ for a in which both candidates are flippable. This is due to the fact minimizing distortion based on an asymmetric distortion table makes it difficult to locate the same relevant candidates for the watermarking image. A symmetric bias table can thus be engaged to solve this problem.

To locate the security concerns, example the oracle attacks, that define to generate the authenticator watermark in Table I and encrypting the XOR ed value of replicated hash value of the binate images recs $>$ and the payload watermark $W_{P}$. To calculate the hash value, the image is divided into 2 parts: 1 ) P1 that contains main contents of the image that contains main contents of the image is used to find out the hash value and 2) P2 that consists of edge portions is used to embed the confidential data. Any tampering conducted will render the computed replicated image. Hash value of the watermaking image $h\left(Y_{w 1}\right)$ differ from the 
reclaimed replicated hash value of the original image $h^{\prime}\left(Y_{1}\right)$ owed to the sensitivity of hash function to alters. Hence, admit the tampering. On the other hand, any tampering conducted to $P 2$ will results in changes either $h^{\prime}\left(Y_{1}\right)$ or $W_{p}^{\prime}$ or both of them. Changes in $h^{\prime}\left(Y_{1}^{\prime}\right)$ finally lead $W_{p}^{\prime} \quad$ to change [see (47)]. Thus, the tampering can be easily detected upon comparison of $W_{p}^{\prime}$ with $W_{p}$. Employing the secure hash function helps detect any changes made to the watermarked document.

\section{COEFFICIENTS COMPARISION}

Fig. 5. Coefficients comparison. (a) Original image. b- m Coarse (b), (f),(j), horizontal detail $(\mathrm{c}),(\mathrm{g}),(\mathrm{k})$, vertical detail $(\mathrm{d}),(\mathrm{h}),(\mathrm{l})$, and diag- onal detail (e),(i),(m) signals obtained from: IMBWT even-even transform b-e, IMBWT odd-odd transform (f)-(i)

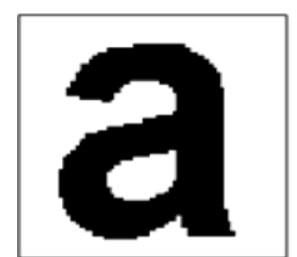

(a)
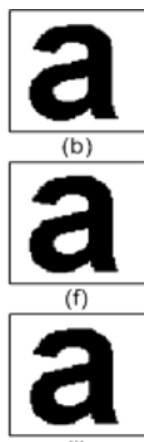

(j)

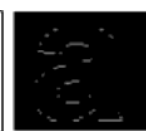

(c)

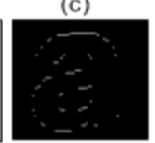

(g)

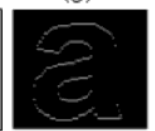

(k)

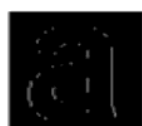

(d)

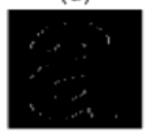

(h)

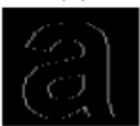

(I)

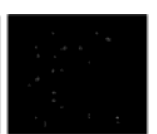

(e)

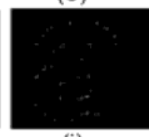

(i)

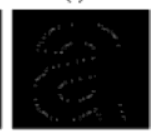

(m)

V.

CONCLUSION

This paper, represents a high-quantity data-veiling scheme for binate images authentication i.e based on the interlaced mor- phological binate wavelet transforms. The concert between the coefficients achieved from different alterations is utilized to indiacte the relevant points for watermark enclose such that blind watermark extraction can be obtained. Two alter cases that are not assemble with one another are employed for orthogonal embedding such a way that not only can the quantity be extremely increased, but also the visual bias can be minimized. Results of comparative ex- periments with other methods emphasize the present scheme's perfection in being able to achieve larger capacity while maintaining adequate visual distortion and low computational cost. Although a conclusion may review the main points of the paper, do not replicate the abstract as the conclusion. A conclusion might elaborate on the importance of the work or suggest applications and extentions.

\section{Acknowledgements}

An acknowledgement section may We wish to acknowledge the help of Mr Chandra Sekahar Assistant Professor, for guidance which helped us work hard towards producing this research project work..

\section{REFERENCES}

\section{Books:}

[1]. J. Cox, M. L. Miller, and J. A. Bloom, Digital Watermarking. San Mateo, CA: Morgan Kaufmann, 2001.

[2]. B. Furht and D. Kirovski, Multimedia Security Handbook, B. Furht and D. Kirovski, Eds. Boca Raton, FL: CRC, 2005.

[3]. Y. Liu, J. Mant, E. Wong, and S. H. Low, "Marking and detection of text documents using transform-domain techniques," in Proc. SPIE, San Jose, CA, 1999, vol. 3657, pp. 317-328.

[4]. Q. Mei, E. K. Wong, and N. Memon, "Data hiding in binary text doc- ument," in Proc. SPIE, 2001, vol. 4314, pp. 369-375.

[5]. Y. C. Tseng and H.-K. Pan, "Data hiding in 2-color images," IEEE Trans. Comput., vol. 51, no. 7, pp. 873-878, Jul. 2002.

[6]. K.-F. Hwang and C.-C. Chang, "A run-length mechanism for hiding data into binary images," in Proc. Pacific Rim Workshop on DigitalSteganography, Kitakyushu, Japan, Jul. 2002, pp. 71-74.

[7]. H. Lu, X. Shi, Y. Q. Shi, A. C. Kot, and L. Chen, "Watermark embedding in DC components of DCT for binary images," in Proc.,IEEE Workshop on Multimedia Signal Processing.

[8]. Ming-Shi Wang and Wei-Che Chen, "Digital image copyright protection scheme based on visual cryptography and singular value decomposition", Optical Engineering,Vol. 46, No. 6, 2007.

[9]. Zhe-Ming Lu, Wei-Min Zheng, Jeng-Shyang Pan and Zhen Sun, "Multipurpose Image Watermarking Method Based on Meanremoved Vector Quantization", Journal of Information Assurance and Security, Vol. 1, pp. 33-42-2006

Chapters in Books:

[10]. Duo Jin, Wei-Qi Yan, Mohan S. Kankanhalli, “ Progressive Color Visual Cryptography”, SPIE Journal of Electronic Imaging (JEI/SPIE) Jan,2006.

Proceedings Papers:

[11]. C. M. Hu and W. G. Tzeng, .Cheating Prevention in VisualCryptography, .IEEE Transaction on Image Processing vol. 16, no. 1, Jan- 2007, pp. 36-45

[12]. M. Wu and B. Liu, "Data hiding in binary images for authentication and annotation," IEEE Trans. Multimedia, vol. 6, no. 4, pp. 528-538,Aug. 2004. 